\title{
ON THE QUASICONVEX EXPOSED POINTS
}

\author{
KEWEI ZHANG ${ }^{1}$
}

\begin{abstract}
The notion of quasiconvex exposed points is introduced for compact sets of matrices, motivated from the variational approach to material microstructures. We apply the notion to give geometric descriptions of the quasiconvex extreme points for a compact set. A weak version of Straszewicz type density theorem in convex analysis is established for quasiconvex extreme points. Some examples are examined by using known explicit quasiconvex functions.
\end{abstract}

Mathematics Subject Classification. 49J45, 49J10, 73V25.

Received April, 1999. Revised August 27, 1999.

\section{INTRODUCTION AND MAIN RESULTS}

In this paper we define quasiconvex exposed points and continue our study on quasiconvex extreme points [35]. We try to determine quasiconvex extreme points by using quasiconvex functions instead of homogeneous Young measures. In other words, we seek geometric descriptions of quasiconvex extreme points, rather than analytical ones. After we prove that the notion of quasiconvex exposed points is a well defined one, that is, it is independent of the generator of quasiconvex set, we establish a Straszewicz type theorem by showing that for a compact set in the space of matrices, the set of quasiconvex exposed points is dense in the set of quasiconvex extreme points. Then we examine the quasiconvex exposed points and quasiconvex extreme points for various examples of known quasiconvex sets or generators of quasiconvex sets used in modelling material microstructures. Finally, we consider the sub-level sets for the explicit quasiconvex relaxation of the squared distance function to a special two point set obtained by Kohn [20]. We study the quasiconvex extreme points of the sub-level sets of this function in details.

Krain-Milman theorem says that a compact convex set is the closed convex hulls of its extreme points [26]. Suppose $K \subset \mathbb{R}^{n}$ is compact and convex, then $K=C\left(K_{e}\right)$ where $K_{e}$ is the set of its extreme points. It is also true that the set $K_{e}$ is the smallest generator of the convex set $K$ in the sense that for every compact $W \subset K$ such that $C(W)=K$, we have $K_{e} \subset W$. In [35] a similar notion for quasiconvex sets was introduced and a Krain-Milman type theorem was established.

A related geometric notion to the extreme points in convex analysis is the so called (convex) exposed points. Straszewicz's theorem says that the set of (convex) exposed points is dense in the set of extreme points [25]. In this paper, we try to explore the possibility of defining a similar notion for quasiconvex hulls and quasiconvex sets. There are several difficulties we encounter if we wish to do so. In convex analysis, hyperplanes and affine functions can be viewed as basic building blocks for convex sets and convex functions [21,25]. However, for quasiconvex sets and quasiconvex functions, it is not clear whether there are simple classes of quasiconvex

Keywords and phrases: Quasiconvex functions, quasiconvex hull, homogeneous Young measure, quasiconvex exposed points, Straszewicz theorem.

1 School of Mathematical Sciences, University of Sussex, Falmer, Brighton BN1 9QH, U.K.; e-mail: k.zhang@sussex.ac.uk 
sets and quasiconvex functions which can form general ones. Intuitively, since one uses affine functions to define convex exposed points, one would think that the proper functions to describe quasiconvex exposed points would be the so called quasi-affine functions (or null Lagrangians), or slightly more complicated, the polyconvex functions [4]. However, since in some situations such as in the case of spaces without rank-one matrices (Th. 1.3), we know that certain simple quasiconvex quadratic functions are not polyconvex [7]. So we need to enlarge the class of allowed "test" functions to all everywhere finite quasiconvex functions. Therefore we define the quasiconvex exposed points in a very general way by using several quasiconvex functions (see Def. 1.3 below). It is then easy to establish a Straszewicz type density result by using quasiconvex exposed points.

For a compact subset $K \subset M^{N \times n}$, we may define its quasiconvex hull $Q(K)$ by cosets of quasiconvex functions [27], or alternatively, by direct quasiconvex relaxations of the distance functions to the set [33]. One way of defining quasiconvex sets is by the sub-level sets of quasiconvex functions, that is, a compact set $K \subset M^{N \times n}$ is quasiconvex if there is a quasiconvex function $f: M^{N \times n} \rightarrow \mathbb{R}$ and a real number $\alpha$ such that

$$
K=\left\{P \in M^{N \times n}, f(P) \leq \alpha\right\} .
$$

The study of quasiconvex hulls and quasiconvex sets is motivated by the variational approach to martensitic phase transitions and material microstructures $[8,9,11,23,29]$. Recently, the notions of quasiconvex sets and quasiconvex hulls have found their applications in the fast developing area of implicit nonlinear systems and general attainment problems [13-17]. A natural question in the study of quasiconvex sets or quasiconvex hulls of a given set is to ask whether there exists a "smallest" subset $K_{0}$ of a quasiconvex set $K$ such that $Q\left(K_{0}\right)=K$. This problem was resolved in [35] where the notion of quasiconvex extreme points was introduced (see Def. 1.2 below).

The following are two equivalent definitions for the quasiconvex hull of a compact set.

Definition 1.1. For a compact subset $K$ of $M^{N \times n}$, the quasiconvex hull $Q(K)$ of $K$ is defined by

$$
Q(K)=\left\{X \in M^{N \times n}, f(X) \leq \sup _{Y \in K} f(Y), \text { for every quasiconvex } f: M^{N \times n} \rightarrow \mathbb{R}\right\}
$$

[29] or equivalently

$$
Q(K)=\left\{A \in M^{N \times n}, Q \operatorname{dist}^{p}(A, K)=0\right\}
$$

[33] for every $p$ with $1 \leq p<\infty$, where $Q \operatorname{dist}^{p}(\cdot, K)$ being the quasiconvexification of the $p$-distance function dist $^{p}(\cdot, K)$ to $K$.

If $Q(K)=K$, then $K$ is called a quasiconvex set.

In [35] we showed that the following set turns out to be the smallest generator of the quasiconvex hull $Q(K)$.

Definition 1.2. (see [35]) Let $K \subset M^{N \times n}$ be non-empty and compact. $P \in K$ is called a quasiconvex extreme point of $K$ if for every gradient homogeneous Young measure $\nu$ supported in $K$ with $\int_{K} \lambda d \nu(\lambda)=P$, then $\nu=\delta_{P}-$ the Dirac mass supported at $P$.

The set of all quasiconvex extreme points of $K$ is denoted by $K_{q, e}$.

The gradient homogeneous Young measure (see Lem. 2.2 below) was defined in [19] based on an approximation lemma in [32], which localizes the oscillation of the sequence of gradients of a bounded sequence in $W^{1, \infty}$. Intuitively, the gradient Young measure represents the oscillation of a weakly (weak-*) convergent sequence while the quasiconvex hull of a set consists of all possible averages of the Young measures supported in the set $[6-9,19]$. Quasiconvex extreme points are those which can only be represented by themselves, that is, by Dirac masses. The definition of quasicovex extreme points by using gradient homogeneous Young measures is an analytic way of describing such points. In the abstract setting for convex sets, Choquet points and the Choquet boundary are defined by using probability measures [1]. However, it is interesting to know whether 
we can find some sort of geometric descriptions of the quasiconvex extreme points. We make an attempt to address this issue in the present paper.

In [35], we established that $K_{q, e}$ is independent of the choice of closed generators of $Q(K)$ and is the smallest generator of $Q(K)$ in the sense that $Q\left(K_{q, e}\right)=Q(K)$, and if $W \subset Q(K)$ is a closed generator of $Q(K)$, then $K_{q, e} \subset W$. We also showed that for the two-well set $K_{2}=S O(n) \cup S O(n) H$ and three-well set $K_{3}=S O(n) \cup S O(n) H_{1} \cup S O(n) H_{2}$ used in the modelling of martensitic phase transformations $\left(K_{2}\right)_{q, e}=K_{2}$, and either $\left(K_{3}\right)_{q, e}=K_{3}$ or $\left(K_{3}\right)_{q, e}$ consists of a subset consists of two wells.

In order to describe quasiconvex extreme points by quasiconvex functions, we need the following definition of quasiconvex exposed points, which is motivated from convex analysis.

Definition 1.3. Let $K \subset M^{N \times n}$ be quasiconvex. We set $K_{0}=K . P \in K$ is called a level- $k$ quasiconvex exposed point of $K$ if there are $k$ compact subsets $K_{1}, K_{2}, \cdots, K_{k}$ such that

$$
K=K_{0} \supsetneq K_{1} \supsetneq K_{2} \supsetneq \cdots \supsetneq K_{k}=\{P\},
$$

and quasiconvex functions $f_{1}, f_{2}, \cdots, f_{k}$ such that

$$
K_{i+1}=\left\{A \in K_{i}, \quad f_{i+1}(A)=\max \left\{f_{i+1}(B), B \in K_{i}\right\}\right\}, \quad i=0,1, \cdots, k-1 .
$$

We denote by $K_{q, k}$ all those quasiconvex exposed points of a compact set $K$ which are of levels less than or equal to $k$. The set of all quasiconvex exposed points of finite levels is denoted by $K_{q \text {,ex }}$, we have $K_{q, \text { ex }}=\cup_{k=1}^{\infty} K_{q, k}$.

For simplicity, we call a quasiconvex exposed point of at most level $k$ as $k_{-}$quasiconvex exposed point. Since $K_{q, k} \subset K_{q, e}$ for all $k \geq 1$ (see Rem. 1.1 below), we see that $K_{q, \text { ex }} \subset K_{q, e}$. We can prove that $\overline{K_{q, \text { ex }}}=\overline{K_{q, e}}$ (see Th. 1.1). In fact, we have a stronger result: $\overline{K_{q, 2}}=\overline{K_{q, e}}$.

Remark 1.1. It is not hard to see that every level $k$ quasiconvex exposed point is a quasiconvex extreme point. In fact, since it is well-known (see, for example $[7,19]$ ) that for everywhere finite quasiconvex function and every gradient homogeneous Young measure, Jensen's inequality holds

$$
\int_{\operatorname{supp} \nu} f(\lambda) d \nu(\lambda) \geq f\left(\int_{\operatorname{supp} \nu} \lambda d \nu(\lambda)\right) .
$$

Now, suppose $P \in K$ is a quasiconvex exposed point of level $k$. Then for every gradient homogeneous Young measure $\nu$ supported in $K=K_{0}$, such that $\int_{\operatorname{supp} \nu} \lambda d \nu(\lambda)=P$, we can prove that $\nu=\delta_{P}$. We proceed as follows. Since

$$
\int_{\operatorname{supp} \nu} f_{1}(\lambda) d \nu(\lambda) \geq f_{1}(P),
$$

and

$$
P \in K_{1}=\left\{A \in K_{0}, f_{1}(A)=\max _{B \in K_{0}} f_{1}(B)\right\},
$$

we see that $\nu$ is supported in $K_{1}$. Similarly, we can show that $\nu$ is supported in $K_{2}$ and so on. Therefore $\nu$ is supported in $K_{k}=\{P\}$. Hence $\nu=\delta_{P}$ and $P \in K_{q, e}$.

In Definition 1.3, we denote by $K_{q, k}$ the set of quasiconvex exposed points up to level $k$ rather than the set of quasiconvex exposed points of exactly level $k$. This is because in general, it is very difficult to prove that a given quasiconvex exposed point is exactly of level $k$, rather than of level $k-1$. The set $K_{q, k}$ for $k=1,2, \ldots$ is designed to estimate how many quasiconvex functions are needed to isolate a quasiconvex extreme point. The problem of finding the minimum number of quasiconvex functions needed to describe a given extreme point seems very difficult at the moment.

In convex analysis, $P$ is an exposed point of a compact convex set $K$ if there is a supporting plane $E$ of $K$ such that $K \cap E=\{P\}$. Therefore there is an affine function $l(\cdot)$, such that $l \leq 0$ in $K$ and the equality 
holds in $K$ only at $P$. This can be viewed as a description of exposed points by using convex functions. This also shows that a convex exposed point is a quasiconvex exposed point of level 1. Hence for every non-empty compact set $K \subset M^{N \times n}, K_{q, 1} \neq \emptyset$.

A natural question concerning Definition 1.3 is whether the level of a quasiconvex exposed point depends on different generator of a quasiconvex set. We have

Proposition 1.1. Let $K \subset M^{N \times n}$ be compact. Then $[Q(K)]_{q, k}=K_{q, k}$.

It was proved in [35] that the extreme points of the convex hull $C(K)$ are also quasiconvex extreme points of $Q(K)$. We have

Proposition 1.2. Let $K \subset M^{n \times N}$ be compact. Then every convex extreme point of $K$ is an Nn_quasiconvex exposed point.

The following is the main result on quasiconvex exposed points for general compact sets in $M^{N \times n}$. It is a weak version of Straszewicz's theorem for convex extreme points.

Theorem 1.1. Let $K \subset M^{N \times n}$ be a compact set and let $P$ be a quasiconvex extreme point of $K$. Then $K$ is either a $2_{-}$quasiconvex exposed point or the limit of a sequence of $2_{-}$quasiconvex exposed points. In other words, $K_{q, 2}$ is dense in $K_{q, e}$.

Remark 1.2. At the moment, I can neither improve Theorem 1.1 to show that $K_{q, 1}$ is dense in $K_{q, e}$ nor prove or disprove that a every quasiconvex extreme is a quasiconvex exposed point of finite level, in other words, I do not know whether $K_{q, e}=K_{q, \mathrm{ex}}$.

If we do not require the quasiconvex functions used in the definition of quasiconvex exposed points to be everywhere finite, it is easy to see that every quasiconvex extreme point is then a quasiconvex exposed point of level 1. In fact, suppose $P_{0} \in K_{q, e}$, we let $B_{n}=\left\{P,\left|P-P_{0}\right|<1 / n\right\}$, we see that $K \backslash B_{n} \neq \emptyset$ for large $n$, say when $n \geq n_{0} \geq 1$. We define the quasiconvex functions $f_{n}(P)=Q \operatorname{dist}^{2}\left(\cdot, K \backslash B_{n}\right)$. Then $f_{n}\left(P_{0}\right)>0$. Otherwise Lemma 2.3 below shows that $P_{0} \notin K_{e}$. Now if we let

$$
f(P)=\sum_{n=n_{0}}^{\infty} \frac{f_{n}(P)}{f_{n}\left(P_{0}\right)},
$$

we see that $f \geq 0$ is quasiconvex, $f(P)<\infty$ if $P \in K, P \neq P_{0}$, and $f\left(P_{0}\right)=+\infty$.

Corollary 1.1. Suppose $K \subset M^{N \times n}$ is a finite set. Then $K_{q, e}=K_{q, 1}$.

Theorem 1.2. Let $f: M^{N \times n} \rightarrow \mathbb{R}$. We assume that

(i) $f$ coercive in the sense that $f(P) \rightarrow+\infty$ if and only if $|P| \rightarrow \infty$;

(ii) $f$ can be written as $f=F+G$, where $G$ is strictly convex (see Def. 2.2) and $F$ is quasiconvex.

For every $\alpha>\inf _{A \in M^{N \times n}} f(A)$, let

$$
K_{\alpha}=\left\{P \in M^{N \times n}, \quad f(P) \leq \alpha\right\}
$$

be the sub-level set of $f$. Then

(1) the set $\left(K_{\alpha}\right)_{q, e}$ of all quasiconvex extreme points is exactly the boundary $\partial K_{\alpha}$ of $K_{\alpha}$, that is

$$
\left(K_{\alpha}\right)_{q, e}=\left\{P \in M^{N \times n}, \quad f(P)=\alpha\right\},
$$

and

(2) every quasiconvex extreme point is a level 1 quasiconvex exposed point, that is

$$
\left(K_{\alpha}\right)_{q, e}=\left(K_{\alpha}\right)_{q, 1}
$$


Corollary 1.2. Let $d(\cdot, \cdot)$ be the Hausdorff distance (see Def. 2.3) on compact sets of $M^{N \times n}$. Suppose K $\subset M^{N \times n}$ is compact and quasiconvex. Then for every $\epsilon>0$, there is a coercive quasiconvex function $f_{\epsilon}$ and some $\alpha>0$, such that the sub-level set

$$
K_{\alpha}=\left\{P \in M^{N \times n}, \quad f(P) \leq \alpha\right\}
$$

contains $K, d\left(K_{\alpha}, K\right) \leq \epsilon$, and

$$
\left(K_{\alpha}\right)_{q, e}=\partial K_{\alpha}=\left\{P \in M^{N \times n}, \quad f(P)=\alpha\right\}
$$

Furthermore $\left(K_{\alpha}\right)_{q, e}=\left(K_{\alpha}\right)_{q, 1}$.

Remark 1.3. The first assertion of Theorem 1.2 is still true under a weaker assumption that $f$ is strictly quasiconvex in the sense that

$$
\int_{M^{N \times n}} f(\lambda) d \nu \geq f(P)
$$

for every $P \in M^{N \times n}$, every homogeneous gradient Young measure $\nu$ supported in $M^{N \times n}$ with $\bar{\nu}=P$, and the equality holds only when $\nu=\delta_{P}$. However, I do not know how to prove the second assertion under the strictly quasiconvexity condition.

Corollary 1.3. Suppose $f: M^{N \times n} \rightarrow R$ is striclty quasiconvex as defined in Remark 1.3 and satisfies (1.3). Suppose

$$
K_{\alpha}=\left\{P \in M^{N \times n}, f(P) \leq \alpha\right\}
$$

is not empty and compact, then

$$
\left(K_{\alpha}\right)_{q, e}=\partial K_{\alpha}=\left\{P \in M^{N \times n}, f(P)=\alpha\right\}
$$

One simple example of uniformly strictly quasiconvex functions is a quadratic form in $M^{N \times n}$ satisfying LegendreHamdamard strong ellipticity condition

$$
f(a \otimes b) \geq c_{0}|a|^{2}|b|^{2}, \quad c_{0}>0
$$

for $a \in \mathbb{R}^{N}, b \in \mathbb{R}^{n}$.

Theorems 1.1, 1.2 and their corollaries can be viewed as some general results for quasiconvex exposed points. Let us now examine some more restricted situations and use various quasiconvex functions to estimate the level of a quasiconvex exposed point.

Let us first consider a compact $K$ contained in a subspace $E$ of $M^{N \times n}$ without rank-one matrices. In this case every point in $K$ is a quasiconvex extreme point. We show that every point in $K$ is in fact a quasiconvex exposed point of level one. We construct a quasiconvex function to achieve this by using a simple rank-one convex quadratic function plus an affine function.

Theorem 1.3. Suppose $E \subset M^{N \times n}$ is a subspace without rank-one matrices. Then for every compact set $K \subset E, Q(K)=K, K_{q, e}=K_{q, \mathrm{ex}}$, and $K_{q, e}=K_{q, 1}$.

The proof of the following result uses a similar method, although the result is much more general in $M^{2 \times 2}$ comparing with Theorem 1.3.

Proposition 1.3. Suppose $K \subset M^{2 \times 2}$ is compact, connected and does not have rank-one connections. Then $K=K_{q, e}=K_{q, 1}$. 
Let us consider the two well set in the modelling of martensitic phase transitions [8,9]. Let $K=S O(n)$ $\cup S O(n) H$. It was established in [35] that $K_{q, e}=K$. We have:

Theorem 1.4. Let $K=S O(n) \cup S O(n) H$, where $S O(n)$ is the set of all rotations in $M^{n \times n}$ with determinant 1 , and $H \in M^{n \times n}$ is positive definite with $H \neq I$, - (the unit matrix). Then $K=K_{q, e}=K_{q, 2}$.

Šverák $[27,28]$ solved the three matrix problem by reducing the problem to the $2 \times 2$ case and by considering the compact subsets of the following

$$
K=\left\{P \in M^{2 \times 2}, P^{T}=P, \operatorname{det} P=1\right\} .
$$

He further showed that the following functions $f_{+}, f_{-}$defined on $M^{2 \times 2}$ are quasiconvex:

$$
f_{ \pm}(P)=\left\{\begin{array}{l}
\operatorname{det} P, \quad P=P^{T}, \quad P \text { is } \pm \text { definite } \\
0, \quad \text { otherwise }
\end{array}\right.
$$

We can establish the following result by using these functions and Theorem 1.3.

Theorem 1.5. Let

$$
L_{+}=\left\{\left(\begin{array}{cc}
x+h & y \\
y & -x+h
\end{array}\right), \quad x, y \in \mathbb{R}\right\}, \quad L_{-}=\left\{\left(\begin{array}{cc}
x-h & y \\
y & -x-h
\end{array}\right), \quad x, y \in \mathbb{R}\right\},
$$

where $h>1$ is a constant. Let

$$
M=\left\{\left(\begin{array}{cc}
x+t & y \\
y & -x+t
\end{array}\right), \quad x, y \in \mathbb{R},-h \leq t \leq h\right\} .
$$

We define $K$ as

Then

$$
K=M \cap\left\{P \in M^{2 \times 2}, P^{T}=P, \operatorname{det} P \geq 1\right\} .
$$

$$
\begin{aligned}
& K_{q, e}=K_{q, 3}=\partial K=\left(M \cap\left\{P \in M^{2 \times 2}, P^{T}=P, \operatorname{det} P=1\right\}\right) \\
& \cup\left[\left(L_{+} \cup L_{-}\right) \cap\left\{P \in M^{2 \times 2}, P^{T}=P, \operatorname{det} P \geq 1\right\}\right] .
\end{aligned}
$$

Remark 1.4. It is easy to see that if we replace the constraint $\operatorname{det} P \geq 1$ by $\operatorname{det} P \geq \alpha$ for any fixed $\alpha>0$, with proper choice of $h$, we can draw the same conclusion. In particular,

$$
(\partial K)_{q, e}=(\partial K)_{q, 2}=\partial K .
$$

We need this remark in the proof of Proposition 1.5 below.

It is easy to see that for a given non-quasiconvex continuous function $f: M^{N \times n} \rightarrow \mathbb{R}$ satisfying

$$
c_{1}|P|^{p}-C_{2} \leq f(P) \leq C_{3}\left(1+|P|^{p}\right),
$$

the quasiconvex relaxation is not strictly quasiconvex. In fact, if at some $P, Q f(P)<f(P)$, there is a nontrivial p-homogeneous Young measure $\nu$ supported in $M^{N \times n}$, such that

$$
\int_{M^{N \times n}} f(\lambda) d \nu(\lambda)=\int_{M^{N \times n}} Q f(\lambda) d \nu(\lambda)=Q f(P) .
$$

Therefore, for the relaxed function $Q f$, if we let

$$
K_{\alpha}=\{P, Q f(P) \leq \alpha\}
$$


be a sub-level set, in general, we have

$$
\left(K_{\alpha}\right)_{q, e} \subsetneq \partial K_{\alpha}
$$

This means some points on the boundary are not quasiconvex extreme point (compare Cor. 1.3). For the quasiconvex relaxtion of the $p$-distnace function $Q$ dist $^{p}(\cdot, K)$, it is interesting to know the location of quasiconvex extreme points of its sub-level sets.

If we consider the convex case, we have a simple formula for convexification of $p$-distance function: $C \operatorname{dist}^{p}(P, K)$ $=\operatorname{dist}^{p}(P, C(K))$. The following result says that we can identify the convex extreme points for a sub-level set $C(K)_{\alpha}$ of the convex relaxation of the $p$-distance function. We identify the set of extreme points by a simple relation which depends only on the information in the sub-level set $K_{\alpha}$ of $\operatorname{dist}^{p}(P, C(K))$. We will establish the result in the case $p=1$. the proof for the general case can be obtained by taking $p$-th root.

Proposition 1.4. Suppose $K \subset \mathbb{R}^{n}$ is compact and satisfies $K=K_{e}$. Let

$$
C \operatorname{dist}^{p}(\cdot, K)=\operatorname{dist}^{p}(\cdot, C(K))
$$

be the convex relaxation of $\operatorname{dist}^{p}(\cdot, K)$ for some real number $p \geq 1$. We define

$$
\begin{aligned}
K_{\alpha} & =\left\{P \in \mathbb{R}^{n}, \operatorname{dist}^{p}(P, K) \leq \alpha\right\} \\
{[C(K)]_{\alpha} } & =\left\{P \in \mathbb{R}^{n}, \operatorname{dist}^{p}(P, C(K)) \leq \alpha\right\}, \\
\tilde{K}_{\alpha} & =\left\{P \in \mathbb{R}^{n}, \operatorname{dist}^{p}(P, K)=\operatorname{dist}^{p}(P, C(K))=\alpha\right\}
\end{aligned}
$$

for $\alpha>0$. Then

$$
\left(C(K)_{\alpha}\right)_{e}=\partial K_{\alpha} \cap \partial C(K)_{\alpha}=\tilde{K}_{\alpha}
$$

This result gives a geometric discription of convex extreme points for $K_{\alpha}$, that is, they are those points $P$ for which the value $\operatorname{dist}^{p}(P, K)$ cannot be pushed down by convex relaxation. However, in the case of quasiconvex relaxation, this is not the case. We will show this as part of the claims of the following explicit calculation of a double well quasiconvex function obtained by Kohn [20]. An interesting geometric feature is that the relaxed functions still maintains the double-well structure.

Proposition 1.5. Let $K=\left\{-\frac{1}{\sqrt{2}} I, \frac{1}{\sqrt{2}} I\right\} \subset M^{2 \times 2}$. Let

$$
F(P)=\operatorname{dist}^{2}(P, K), \quad f(P)=Q F(P) \quad P \in M^{2 \times 2},
$$

where $f(P)$ is explicitly given by [20] (for a much more general case) as

$$
f(P)=\min _{0 \leq \theta \leq 1}\left(|P-(1-2 \theta) I|^{2}+4 \theta(1-\theta)\right) .
$$

Let

$$
E_{1}=\left(\begin{array}{cc}
\frac{1}{\sqrt{2}} & 0 \\
0 & \frac{1}{\sqrt{2}}
\end{array}\right), \quad E_{2}=\left(\begin{array}{cc}
0 & \frac{1}{\sqrt{2}} \\
-\frac{1}{\sqrt{2}} & 0
\end{array}\right), \quad E_{3}=\left(\begin{array}{cc}
\frac{1}{\sqrt{2}} & 0 \\
0 & \frac{-1}{\sqrt{2}}
\end{array}\right), \quad E_{4}=\left(\begin{array}{cc}
0 & \frac{1}{\sqrt{2}} \\
\frac{1}{\sqrt{2}} & 0
\end{array}\right) .
$$

Then $\left\{E_{i}, i=1, \ldots 4\right\}$ forms an orthonormal basis in $M^{2 \times 2}$. We write a general element in $M^{2 \times 2}$ as

$$
P=t E_{1}+s E_{2}+x E_{3}+y E_{4}, \quad(t, s, x, y) \in \mathbb{R}^{4},
$$

and let $P_{0}=t_{0} E_{1}+s_{0} E_{2}+x_{0} E_{3}+y_{0} E_{4}$. Let

$$
K_{\alpha}=\left\{P \in M^{2 \times 2}, f(P) \leq \alpha\right\},
$$


for $\alpha \geq 0$, then,

(1) $P_{0} \in\left[K_{f\left(P_{0}\right)}\right]_{q, 3}$ if $P_{0} \in M_{1}=\left\{P_{0},\left|t_{0}\right| \geq 1 / 2\right\}$;

(2) $P_{0} \in\left[K_{f\left(P_{0}\right)}\right]_{q, 4}$ if $P_{0} \in M_{2}=\left\{P_{0},\left|t_{0}\right|<1 / 2, t_{0}^{2}>x_{0}^{2}+y_{0}^{2}\right\}$;

(3) $P_{0} \notin\left[K_{f\left(P_{0}\right)}\right]_{q, e}$ if $P_{0} \in M_{3}=\left\{P_{0},\left|t_{0}\right|<1 / 2, t_{0}^{2} \leq x_{0}^{2}+y_{0}^{2}\right\}$.

Moreover,

(4) $K_{\alpha} \subset M_{1}$ if $\alpha \leq 1 / 4$,

(5) $K_{\alpha} \cap M_{k} \neq \emptyset$, for $k=1,2$, if $\alpha>1 / 4 ; K_{\alpha} \subset M_{1} \cup M_{2}$ if $\alpha<1 / 2$, and

$$
M_{3} \subset\{P, f(P) \geq 1 / 2\}, \quad M_{3} \cap K_{\alpha} \neq \emptyset \text { if } \alpha \geq 1 / 2
$$

(6) in $M_{2} \cup M_{3}, Q \operatorname{dist}^{2}\left(P_{0}, K\right)<\operatorname{dist}^{2}\left(P_{0}, K\right)$.

In the proof of Proposition 1.5, we will see that points in $M_{2}$ are in the relaxed set where $f(P)<F(P)$ and they are also quasiconvex extreme points as stated in (2) above. Therefore, if we let

$$
\begin{aligned}
\tilde{K}_{\alpha} & =\left\{P \in M^{N \times n}, \operatorname{dist}^{p}(P, K)=Q \operatorname{dist}^{p}(P, K)=\alpha\right\}, \\
K_{\alpha} & =\left\{P \in M^{N \times n}, \operatorname{dist}^{p}(P, K) \leq \alpha\right\}, \\
\partial K_{\alpha} & =\left\{P \in M^{N \times n}, \operatorname{Qdist}^{p}(P, K)=\alpha\right\} .
\end{aligned}
$$

Then, in general

$$
Q\left(\tilde{K}_{\alpha}\right) \subsetneq Q\left(\partial K_{\alpha}\right)=K_{\alpha}
$$

Therefore, a similar statemet as Proposition 1.4 is not true in general for quasiconvex relaxations of the $p$-distance function.

An interesting feature of Part (6) is that the level set $\partial K_{\alpha}$ is the set of quasiconvex extreme points only when $\alpha<1 / 2$ while $\alpha=2$ is the critical value of $f$ with critical point 0 , where the level sets change their topological type. When $\alpha>1 / 2, \partial K \alpha$ is homeomorphic to a sphere. When $\alpha<1 / 2$, it is a union of two disjoint sphere.

\section{Preliminary Results}

We denote by $M^{N \times n}$ the space of all real $N \times n$ matrices with $\mathbb{R}^{N n}$ norm. If $E \subset M^{N \times n}$ is a linear subspace, we write $P_{E}$ and $P_{E \perp}$ as the orthogonal projections from $M^{N \times n}$ to $E$ and its orthogonal complement $E^{\perp}$ respectively. meas $(U)$ is the Lebesgue measure of a measurable subset $U \subset \mathbb{R}^{n}$ and

$$
\operatorname{dist}(Q, K)=\inf _{P \in K}|Q-P|
$$

denotes the distance function from a point $Q \in M^{N \times n}$ to a set $K \subset M^{N \times n}$. From now on $\Omega$ denotes a non-empty, open and bounded subset of $\mathbb{R}^{n}$. For a given set $K \subset \mathbb{R}^{s}$, int $K, \dot{\bar{K}}$ and $\partial K$ denote its interior, closure and boundary. We denote by $D u$ the gradient of a (vector-valued) function $u$ and we define the space $C_{0}^{k}\left(\Omega, \mathbb{R}^{N}\right)$, the $L^{p}$ spaces and Sobolev spaces $W^{1, p}$ in the usual way. We say that $K \subset M^{N \times n}$ has a rank-one connection if there exist $A, B \in K$ such that $\operatorname{rank}(A-B)=1$. The support of a measure $\nu$ is denoted by $\operatorname{supp} \nu$, and let $\bar{\nu}=\int_{\text {supp }} \lambda d \nu(\lambda)$.

Let $f: M^{N \times n} \rightarrow \mathbb{R}$ be a continuous function. $f$ is quasiconvex $(c f .[4,12,22])$ in $M^{N \times n}$ if for every open and bounded subset $\Omega$ of $\mathbb{R}^{n}$, every $P \in M^{N \times n}$ and every $\phi \in C_{0}^{\infty}\left(\Omega, \mathbb{R}^{N}\right)$,

$$
\int_{\Omega} f(P+D \phi(x)) d x \geq \int_{\Omega} f(P) d x .
$$

The class of quasiconvex functions is independent of the choice of $\Omega$. It is well known now that $I(u)=\int_{\Omega} f(D u) d x$ is lower semicontinuous in the Sobolev space $W^{1, \infty}\left(\Omega, \mathbb{R}^{N}\right)$, in the weak-* sense if and only if $f$ is quasiconvex 
(see [2,4,22]). Suppose in addition, $f$ satisfies $0 \leq f(P) \leq C\left(1+|P|^{p}\right)$ for $P \in M^{N \times n}$, for some constants $C>0$ and $p \geq 1$, then $I(\cdot)$ is weakly lower semicontinuous in $W^{1, p}\left(\Omega, \mathbb{R}^{N}\right)$ if and only if $f$ is quasiconvex (see [2] for the general statements and proofs).

For a given function, we can consider its quasiconvexification (quasiconvex relaxation):

Definition 2.1. [12] Suppose $f: M^{N \times n} \rightarrow \mathbb{R}$ is continuous. The quasiconvexification of $f$ is defined by

$$
\sup \{g \leq f ; g \text { quasiconvex }\}
$$

and will be denoted by $Q f$.

Proposition 2.2. (see [12]) Suppose $f: M^{N \times n} \rightarrow \mathbb{R}$ is continuous, then

$$
Q f(P)=\inf _{\phi \in C_{0}^{\infty}\left(\Omega ; \mathbb{R}^{N}\right)} \frac{1}{\operatorname{meas}(\Omega)} \int_{\Omega} f(P+D \phi(x)) d x,
$$

where $\Omega \subset \mathbb{R}^{n}$ is a bounded domain. Qf is quasiconvex. In particular the infimum in (2.1) is independent of the choice of $\Omega$.

In the variational approach to martensitic phase transitions, the integrand $f$ is sometimes in a special form where $f \geq 0$, and $f(P)=0$ if and only if $P \in K$, where $K \subset M^{N \times n}$ is a compact set (see [7-9,11]). Such functions are in general not quasiconvex. Suppose $\left(u_{j}\right)$ is a bounded sequence in the Sobolev space $W^{1, p}\left(\Omega, \mathbb{R}^{N}\right)$, such that $I\left(u_{j}\right) \rightarrow 0$, as $j \rightarrow \infty$, we are interested in the oscillating behaviour of the sequence $\left(D u_{j}\right)$ and the possible "microstructures" it may generate. The notion of Young measures is a useful way of describing microstructures.

Lemma 2.1 (Young measures). (See, for example [5,31].) Suppose $\left(U_{k}\right)$ is a bounded sequence in $L^{\infty}\left(\Omega ; R^{s}\right)$, and for some compact set $K \subset R^{s}$, meas $\left(\left\{x \in \Omega: U_{k}(x) \notin G\right\}\right) \rightarrow 0$ as $k \rightarrow \infty$ for every open set $G \supset K$. Then there exists a subsequence (still denoted by $U_{k}$ ) and an associated family of probability measures $\nu_{x}$ on $R^{s}$ such that (i) $\nu_{x}$ is supported on $K$ for almost every $x \in \Omega$; (ii) for any continuous function $\psi$ on $R^{s}, \psi\left(U_{k}\right)$ converges in the weak-* sense to the function $x \rightarrow \int_{R^{s}} \psi(\lambda) d \nu_{x}(\lambda)$.

In the above definition, if the sequence $U_{k}$ has the form $U_{k}=D u_{k}$, where $\Omega \subset \mathbb{R}^{n}$ is open and bounded, and $\left(u_{k}\right)$ is a bounded sequence in $W^{1, \infty}\left(\Omega, \mathbb{R}^{s}\right)$, then the corresponding Young measure $\nu_{x}$ is called Young measure limit of gradients or gradient Young measure (see $[7,19])$. The Young measure is trivial if $\nu_{x}$ is a Dirac measure for a.e. $x$. In this case there exists a function $u$ such that $\nu_{x}$ is the Dirac measure at $D u(x)$, and up to a subsequence, $D u_{k} \rightarrow D u$ almost everywhere. In general, the Young measure may be nontrivial.

One of the restrictions of Young measure limit of gradients is that for every quasiconvex function $f: M^{N \times n}$ $\rightarrow \mathbb{R}$

$$
\int_{\operatorname{supp} \nu_{x}} f(\lambda) d \nu_{x} \geq f\left(\int_{\operatorname{supp} \nu_{x}} \lambda d \nu_{x}\right)
$$

for almost every $x \in \Omega$ (see for example $[7,10,19]$ ).

Lemma 2.2 (gradient homogeneous Young measures). (See [19] for a more general statement.) Let $\left\{\nu_{x}\right\}_{x \in \Omega}$ be a family of gradient Young measures $\left\{\nu_{x}\right\}_{x \in \Omega}$ with bounded supports, that is $\operatorname{supp} \nu_{x} \subset K$, where $K$ is a compact subset of $M^{N \times n}$ and $\int_{K} \lambda d \nu_{x_{0}}(\lambda)=P_{0}$ for almost every $x_{0} \in \Omega$. Then for almost every $x_{0} \in \Omega$, there exists a bounded sequence $\left(\phi_{k}\right)$ in $W_{0}^{1, \infty}\left(D, \mathbb{R}^{N}\right)$ such that the corresponding family of gradient Young measures $\left\{\bar{\nu}_{y}\right\}$ of the squence $\left(P_{0}+D \phi_{k}\right)$ satisfy $\bar{\nu}_{y}=\nu_{x_{0}}$ for almost every $y \in D$, where $D$ is the unit open hypercube in $\mathbb{R}^{n} . \bar{\nu}_{y}$ is called a gradient homogeneous Young measure.

From now on, we mean by homogeneous Young measures as gradient homogeneous Young measure (we write HYM for simplicity) by $\nu$ and write its integral average on its compact support $K$ as $\bar{\nu}_{K}:=\int_{K} \lambda d \nu(\lambda)$.

The following result ([35], Lem. 3.1) is useful for describing the quasiconvex hull of a compact set. 
Lemma 2.3. Suppose $K \subset M^{N \times n}$ is compact and let $P \in Q(K)$. Then there exists a HYM $\nu$ supported in $K$ such that $\bar{\nu}_{K}=P$. In particular, when $P \in Q(K) \backslash K, \nu \neq \delta_{P}$.

The following is a result for quasiconvex hulls of sets contained in a plane without rank-one connections. It is a consequence of [7] (Th. 4.1). For the proof, see [33].

Proposition 2.3. Suppose $K \subset E \subset M^{N \times n}$ is compact. Then

$$
Q(K)=K
$$

Definition 2.2. A convex function $G: \mathbb{R}^{m} \rightarrow R$ is called strictly convex at $x_{0}$ there is an affine function $g: \mathbb{R}^{n} \rightarrow \mathbb{R}$, such that $G(y) \geq g(y)$ for all $y \in \mathbb{R}^{m}$, and the equality holds only when $y=x_{0}$. A convex function $G: \mathbb{R}^{m} \rightarrow R$ is called strictly convex if it is strictly convex at every point $x \in \mathbb{R}^{n}$.

We conclude this section by giving the definition of the Hausdorff metric.

Definition 2.3. Suppose $K, S \subset \mathbb{R}^{n}$ are nonempty compact sets. The Hausdorff metric between $K$ and $S$ is defined by

$$
d(K, S)=\inf \left\{\delta>0, K \subset S_{\delta}, S \subset K_{\delta}\right\},
$$

where $K_{\delta}$ and $S_{\delta}$ are the closed $\delta$-neighbourhoods of $K$ and $S$ respectively (see [18]).

\section{Proofs of the MAIN RESUlts}

In this section, we prove our main results.

Proof of Proposition 1.1. Let $P_{0} \in K_{q, k}$. By definition, we have

$$
K \supsetneq K_{1} \supsetneq K_{2} \supsetneq \cdots \supsetneq K_{s}=\left\{P_{0}\right\}
$$

for some $s$ with $1 \leq s \leq k$.

$$
K_{1}=\left\{A \in K, f_{1}(A)=\sup _{X \in K} f_{1}(X)\right\} .
$$

From Definition 1.1 (1.1), we see that

$$
\sup _{X \in K} f_{1}(X)=\sup _{X \in Q(K)} f_{1}(X)
$$

Let

$$
\tilde{K}_{1}=\left\{A \in K, f_{1}(A)=\sup _{X \in Q(K)} f_{1}(X)\right\},
$$

we see that $K_{1} \subset \tilde{K}_{1}$ and $P_{0} \in \tilde{K}_{1}$. Now we show that $\tilde{K}_{1} \subset Q\left(K_{1}\right)$ so that the proof can go further. If the claim was not true, there is some $P_{1} \in \tilde{K}_{1} \backslash Q\left(K_{1}\right)$. Let us then consider the set

$$
W=\left\{Y \in \tilde{K}_{1}, Q \operatorname{dist}\left(Y, Q\left(K_{1}\right)\right)=\sup _{X \in \tilde{K}_{1}} Q \operatorname{dist}\left(X, Q\left(K_{1}\right)\right)>0\right\} .
$$

Noticing that $Q \operatorname{dist}\left(X, Q\left(K_{1}\right)\right)=0$ if and only if $X \in Q\left(K_{1}\right)$, we see that $W \cap \tilde{K}_{1}=\emptyset$. Let $P_{2} \in W$ be a quasiconvex extreme point, we see that $P_{2} \in[Q(K)]_{q, e}$, hence $P_{2} \in K_{q, e}$. Thus $P_{2} \in K_{1} \subset \tilde{K}_{1}$. This is a contradiction.

Now on $K_{1}$, we have

$$
P_{0} \in K_{2}=\left\{A \in K_{1}, f_{2}(A)=\sup _{X \in K_{1}} f_{2}(X)\right\}
$$


and

$$
\sup _{X \in K_{1}} f(X)=\sup _{X \in \tilde{K}_{1}} f(X)=\sup _{X \in Q\left(K_{1}\right)} f(X) .
$$

Let

$$
\tilde{K}_{2}=\left\{A \in \tilde{K}_{1}, f(A)=\sup _{X \in \tilde{K}_{1}} f(X)\right\} .
$$

If we repeat the argument in the previous step, we see that

$$
K_{2} \subset \tilde{K}_{2} \subset Q\left(K_{2}\right) .
$$

Hence inductively, we may find $\tilde{K}_{i}$ with $i=1,2, \ldots, s$, such that

$$
Q(K) \supset \tilde{K}_{1} \supset \tilde{K}_{2} \supset \cdots \supset \tilde{K}_{s}
$$

with $K_{i} \subset \tilde{K}_{i} \subset Q\left(K_{i}\right)$ and

$$
\tilde{K}_{i}=\left\{A \in \tilde{K}_{i-1}, f_{i}(A)=\sup _{X \in \tilde{K}_{i-1}} f(X)\right\},
$$

for $i=1,2, \ldots, s$. Here we set $\tilde{K}_{0}=Q(K)$. Now since $K_{s}=\left\{P_{0}\right\}$, we have $Q\left(K_{s}\right)=K_{s}$ so that $\tilde{K}_{s}=\left\{P_{0}\right\}$. Hence $P_{0} \in[Q(K)]_{q, s} \subset[Q(K)]_{q, k}$. Therefore $K_{q, k} \subset[Q(K)]_{q, k}$.

Let us now prove the other inclusion $[Q(K)]_{q, k} \subset K_{q, k}$. Let $P_{0} \in[Q(K)]_{q, k}$. From the definition of quasiconvex exposed points, we see that $P_{0}$ is a quasiconvex extreme point of $Q(K), P_{0} \in[Q(K)]_{q, e}$ so that $P_{0} \in K_{q, e} \subset K$. From the Definition 1.3, there are

$$
Q(K)=\tilde{K}_{0} \supsetneq \tilde{K}_{1} \supsetneq \tilde{K}_{2} \supsetneq \cdots \supsetneq \tilde{K}_{s}=\left\{P_{0}\right\},
$$

for some $s$ with $1 \leq s \leq k$, such that

$$
\tilde{K}_{i}=\left\{A \in \tilde{K}_{i-1}, f_{i}(A)=\sup _{X \in \tilde{K}_{i-1}} f(X)\right\},
$$

for $i=1,2, \ldots, s$ with $f_{i}$ quasiconvex. Now we define $K_{0}=K$,

$$
K_{i}=\left\{A \in K_{i-1}, f_{i}(A)=\sup _{X \in K_{i-1}} f(X)\right\} .
$$

As in the proof of the previous part, we can easily see that $K_{i} \subset \tilde{K}_{i} \subset Q\left(K_{i}\right)$ and we also have

$$
\sup _{X \in K_{i-1}} f(X)=\sup _{X \in \tilde{K}_{i-1}} f(X)=\sup _{X \in Q\left(K_{i-1}\right)} f(X),
$$

and

$$
K=K_{0} \supset K_{1} \supset K_{2} \supset \cdots \supset K_{s}=\left\{P_{0}\right\}
$$

The proof is complete.

Proof of Proposition 1.2. Let $P \in K_{e}$. We can use at most $N n$ affine functions to isolate $P \in K_{e}$ as follows. Let $E_{1}$ be a supporting plane of $C(K)$ [25], passing through $P$. Let $K_{1}=C(K) \cap E_{1}$. There exists an affine function $l_{1}(\cdot)$, such that $l_{1}(A) \leq 0$ in $K$ and the equality holds only when $A \in K_{1}$. Obviously, $P \in K_{1}$. If $K_{1}=\{P\}$, then $P$ is a convex exposed point and the proof is finished. If $K_{1} \neq\{P\}$, we may repeat the previous 
argument to obtain $K_{2}, l_{2}(\cdot)$ and so on. Since the dimension of $C(K)$ is less than or equal to $N n$, we need at most $N n$ steps.

Proof of Theorem 1.1. Suppose $P \in\left(K_{q, e} \backslash K_{q, 2}\right)$. Then for every $\epsilon>0$, we seek to prove that there is $P^{\prime} \in K_{q, 2}$, such that $\left|P-P^{\prime}\right|<\epsilon$. Let $B(P, \epsilon)$ be the open ball in $M^{N \times n}$ centred at $P$ with radius $\epsilon$. Set $K(\epsilon)=K \backslash B(P, \epsilon)$. Now let us consider the quasiconvex function $f_{1}(A)=Q \operatorname{dist}(A, K(\epsilon)), A \in M^{N \times n}$. We claim that $f_{1}(P)>0$. Otherwise $Q \operatorname{dist}\left(P, K_{\epsilon}\right)=0$. Lemma 2.3 implies that there is a HYM $\nu$ supported in $K(\epsilon)$ such that $\bar{\nu}=P$. Obviously $\nu \neq \delta_{P}$. This contradicts to the fact that $P \in K_{q, e}$. Now let

$$
K_{1}=\{A \in K, Q \operatorname{dist}(A, K(\epsilon))=\max \{Q \operatorname{dist}(P, K(\epsilon)), P \in K\}\}
$$

then $K_{1} \subset B(P, \epsilon), K_{1} \cap K(\epsilon)=\emptyset$. If $K_{1}$ consists only one point $B$, then $B \neq P$ because $B \in K_{q, 1} \subset K_{q, 2}$ by definition. So let $P^{\prime}=B$, we have $P^{\prime} \in K_{q, 2}$ and $\left|P-P^{\prime}\right|<\epsilon$. If $K_{1}$ consists of more than one point, we may claim that there is an convex exposed point $P^{\prime} \neq P$ of $K_{1}$. Since $P^{\prime} \in\left(K_{1}\right)_{q, 1}$, we see that $P^{\prime} \in K_{q, 2}$ and $\left|P-P^{\prime}\right|<\epsilon$. The prove is complete.

Proof of Corollary 1.1. We only need to prove that $K_{q, e} \subset K_{q, 1}$. For $P_{0} \in K_{q, e}$, as in the proof of Theorem 1.1, we consider the quasiconvex function $f(A)=Q \operatorname{dist}\left(A, K \backslash\left\{P_{0}\right\}\right)$ defined for $A \in M^{N \times n}$. Because $K$ is finite, we claim that $f\left(P_{0}\right)>0$. Otherwise $P_{0} \notin K_{q, e}$. Since $f(A)=0$ when $A \in K \backslash\left\{P_{0}\right\}$, we see that $P_{0} \in K_{q, 1}$.

Proof of Theorem 1.2. It is easy to see that $\left(K_{\alpha}\right)_{q, e} \subset \partial K_{\alpha}$ because for every interior point $P$ of $K$, there is a rank-one matrix $R$, and two numbers $t_{1}>0$ and $t_{2}<0$ such that $A=P+t_{1} R \in \partial K_{\alpha}, B=P+t_{2} R \in \partial K_{\alpha}$. We see that $\operatorname{rank}(A-B)=1$ and $P$ is a convex combination of $A$ and $B$. Therefore $P$ is not a quasiconvex extreme point.

Now we prove that $\partial K_{\alpha} \subset\left(K_{\alpha}\right)_{q, 1}$ and reach our conclusions. Let $A \in \partial K_{\alpha}$. From the definition of $K_{\alpha}$, we see that $f(A)=\alpha$. Since $f=F+G$ with $F$ quasiconvex and $G$ strictly convex, by Definition 2.2 , there is an affine function $g: M^{N \times n} \rightarrow \mathbb{R}$, such that $G(P) \geq g(P)$ for all $P \in M^{N \times n}$ and the equality holds only when $P=A$. Now let $f_{1}(P)=g(P)+F(P)$ for all $P \in M^{N \times n}$. It is obvious that $f_{1}$ is quasiconvex, $f_{1}(P) \leq f(P)$ and the equality holds only when $P=A$. Now, we check that $A$ is the only maximum point of $f_{1}$ in $K_{\alpha}$. Suppose $B \in K_{\alpha}, B \neq A$ we have $f(B) \leq \alpha$, so that

$$
\alpha=f_{1}(A)=f(A) \geq f(B)>f_{1}(B) .
$$

By definition, $A \in\left(K_{\alpha}\right)_{q, 1}$. The proof is complete.

Proof of Corollary 1.2. We define $f(P)=Q \operatorname{dist}(P, K)$ and $f_{\delta}=\delta|P|^{2}+f(P)$ for $P \in M^{N \times n}$. Since $K$ is quasiconvex, we have that $f(P)=0$ if and only if $P \in K$. Setting $a=\max \left\{|P|^{2}, P \in K\right\}$, we see that

$$
\max \left\{f_{\delta}(P), P \in K\right\}=a \delta
$$

Now we define

$$
K_{a \delta}=\left\{P \in M^{N \times n}, f_{\delta} \leq a \delta\right\} .
$$

It is easily seen that $K \subset K_{a \delta}$. Since $K$ is compact and $f$ is continuous, we can easily prove that $d\left(K, K_{a \delta}\right) \rightarrow 0$ as $\delta \rightarrow 0_{+}$. Therefore for any $\epsilon>0$, there is a $\delta_{0}>0$, such that $d\left(K, K_{a}\right)<\epsilon$ when $0<\delta<\delta_{0}$. It is easy to see that $f_{\delta}$ satisfies the assumptions of Theorem 1.2 , for every $\delta>0$, hence

$$
\left(K_{a \delta}\right)_{q, e}=\partial K_{a \delta}=\left(K_{a \delta}\right)_{q, 1} .
$$

The proof is complete if we restrict $\delta$ to satisfy $0<\delta<\delta_{0}$. 
Proof of Corollary 1.3. As in the proof of Theorem 1.2, we see that $\left(K_{\alpha}\right)_{q, e} \subset \partial\left(K_{\alpha}\right)$. Let $P \in \partial\left(K_{\alpha}\right)$, and let $\nu$ be a homogeneous Young measure supported in $K_{\alpha}$ with $\bar{\nu}=P$. Since $f(P)=\alpha$ and for every $A \in K_{\alpha}$, we have $f(A) \leq \alpha$. Hence $\nu \subset \partial\left(K_{\alpha}\right)$. Now we have

$$
\int_{\partial\left(K_{\alpha}\right)} f(\lambda) d \nu(\lambda)=f(P) .
$$

Now we use the strict quasiconvexity property of $f$ to conclude that $\nu=\delta_{P}$.

Proof of Theorem 1.3. We define a quasiconvex function to reach our conclusion. Let $P_{0} \in K \subset E$, where $E \subset M^{N \times n}$ is a subspace without rank-one matrices. Let $E^{\perp}$ be its orthogonal complement and let $P_{E}$ and $P_{E^{\perp}}$ be the orthogonal projection on $E$ and $E^{\perp}$ respectively. We define

$$
\alpha_{E}=\min \left\{\left|P_{E^{\perp}}(a \otimes b)\right|^{2}, \quad a \in \mathbb{R}^{N},|a|=1, b \in \mathbb{R}^{n},|b|=1\right\} .
$$

Then $\alpha_{E}>0$. Now we define a function on $E$ as $F(B)=-\alpha_{E}\left|B-P_{0}\right|^{2}$ for $B \in E$ and let

$$
f(A)=F\left(P_{E}(A)\right)+\left|P_{E^{\perp}}(A)\right|^{2}
$$

for $A \in M^{N \times n}$. We see that on $E, f=F$ and $F(\cdot)$ reaches its maximum 0 in $E$ only at $P_{0}$. In order to show that $P_{0} \in K_{q, 1}$, we only need to prove that $f$ is quasiconvex. We have

$$
\begin{aligned}
& f(A)=-\alpha_{E}\left|P_{E}(A)-P_{0}\right|^{2}+\left|P_{E^{\perp}}(A)\right|^{2}=\left[\left|P_{E^{\perp}}(A)\right|^{2}-\alpha_{E}\left|P_{E}(A)\right|^{2}\right]+\left[2 \alpha_{E}\left(P_{E}(A) \cdot P_{0}\right)-\alpha_{E}\left|P_{0}\right|^{2}\right] \\
& :=H(A)+g(A)
\end{aligned}
$$

where

$$
\begin{aligned}
H(A) & =\left|P_{E^{\perp}}(A)\right|^{2}-\alpha_{E}\left|P_{E}(A)\right|^{2} \\
g(A) & =2 \alpha_{E}\left(P_{E}(A) \cdot P_{0}\right)-\alpha_{E}\left|P_{0}\right|^{2} .
\end{aligned}
$$

Since $g(A)$ is affine in $A$, it is quasiconvex. Since $H(A)$ is a quadratic form in $A$, to show that it is quasiconvex, we only need to show that it satisfies the Legendre-Hamdamard ellipticity condition $H(a \otimes b) \geq 0$ for all $a \in \mathbb{R}^{N}$, $b \in \mathbb{R}^{n}$. we have

$$
\begin{aligned}
H(a \otimes b) & =\left|P_{E^{\perp}}(a \otimes b)\right|^{2}-\alpha_{E}\left|P_{E}(a \otimes b)\right|^{2} \geq \alpha_{E}|a|^{2}|b|^{2}-\alpha_{E}\left|P_{E}(a \otimes b)\right|^{2} \\
& =\alpha_{E} \mid\left(\left.a \otimes b\right|^{2}-\alpha_{E}\left|P_{E}(a \otimes b)\right|^{2} \geq 0 .\right.
\end{aligned}
$$

Therefore $H(\cdot)$ is quasiconvex, so that $f(\cdot)$ is quasiconvex. Thus $P_{0} \in K_{q, 1}$. The proof is finished.

When $K \subset M^{2 \times 2}$ is connected and does not have rank-one connections, Šverák [30] observed that $\operatorname{det}(A$ $-B)>0$ for all $A, B \in K, A \neq B$ or $\operatorname{det}(A-B)<0$ for all $A, B \in K, A \neq B$ respectively. He used this observation to prove Tartar's conjecture in the $M^{2 \times 2}$ case. Among his results, he established that if we use the notion of quasiconvex extreme point, $K=K_{q, e}$. It was established in [33] that this type of sets are Lipschitz graphs on a two dimension subspace.

Proof of Proposition 1.3. From [30], we see that either (i) $\operatorname{det}(A-B)>0$ for all $A, B \in K, A \neq B$ or (ii) $\operatorname{det}(A-B)<0$ for all $A, B \in K, A \neq B$. Let us fix $P_{0} \in K$ and consider

$$
f(A)=-\operatorname{det}\left(A-P_{0}\right), \quad A \in M^{N \times n}
$$

when (i) happens, or

$$
f(A)=\operatorname{det}\left(A-P_{0}\right), \quad A \in M^{N \times n}
$$


when (ii) happens. In both cases, we have that $f$ is quasiconvex and $f(A) \leq 0$ when $A \in K$. The equality holds only when $A=P_{0}$. Therefore $P_{0} \subset K_{q, 1}$. The conclusion follows.

Before we prove Theorem 1.4, let us examine the set $S O(n)$, it is well known and easy to prove that $S O(n)$ is a strict convex surface. The tangent plane at any point $P$ on $S O(n)$ touches the set at $P$ only. Therefore every point in $S O(n)$ is a convex exposed point.

Proof of Theorem 1.4. It is known [35] that $Q \operatorname{dist}^{2}(\cdot, S O(n))$ and $Q \operatorname{dist}^{2}(\cdot, S O(n) H)$ are both left invariant and vanishes on $S O(n)$ and $S O(n) H$ respectively. For every $P \in S O(n) H$, we have

$$
Q \operatorname{dist}^{2}(P, S O(n))=Q \operatorname{dist}^{2}(H, S O(n)):=\alpha_{1}>0
$$

which is independent of $P$. Therefore, if $\nu$ is a homogeneous Young measures supported in $K$, such that $\bar{\nu}=P \in S O(n) H$, we have $\operatorname{supp} \nu \subset S O(n) H$. Since $S O(n) H$ is also strictly convex, $P$ is a convex exposed point of $C(S O(n) H)$. Therefore there is a affine function $l(\cdot)$ such that $l(A) \leq 0$ in $C(S O(n) H)$ and the equality holds only when $A=P$. Hence $P \in K_{q, 2}$. A similar argument as above gives that every $A \in S O(n)$ is contained in $K_{q, 2}$ as well.

Obviously, the convex exposed points of $K=S O(n) \cup S O(n) H$ belong to $K_{q, 1}$. Theorem 4.1 gives a description of general quasiconvex extreme points by means of quasiconvex exposed points. I do not know whether $K_{q, e}=K_{q, 1}$.

Proof of Theorem 1.5. The set $K$ can be written as the union of two disjoint sets $K=K_{+} \cup K_{-}$, where

$$
K_{+}=\{P \in K, P \text { is positive definite }\} \text {, and } K_{-}=\{P \in K, P \text { is negative definite }\}
$$

Also if we consider $K$ as a set in the space $S_{2}$ of $2 \times 2$ symmetric matrices, then $K$ is the closure of its relative interior.

We first show that the quasiconvex extreme points are contained in the relative boundary of $K$ in $S_{2}$, that is

$$
K_{q, e} \subset \partial K=\{P \in K, \operatorname{det} P=1,\} \cup\left[K \cap\left(L_{+} \cup L_{-}\right)\right] .
$$

Since $S_{2}$ contains rank-one matrices, for example $A_{0}=\operatorname{diag}(1,0)$, if $P$ is an interior point relative to $S_{2}$, we can find $t_{1}>0, t_{2}<0$, such that $P+t_{1} A_{0} \in \partial K, P+t_{2} A_{0} \in \partial K$, so that $P$ is a convex combination of two rank-one connected matrices. Hence $P \notin K_{q, e}$.

Now we prove that $K \cap\left(L_{+} \cup L_{-}\right) \subset K_{q, 2}$. Since $L_{+}$and $L_{-}$are both supporting planes of $C(K)$. If $P_{0} \in K \cap L_{+}$, there is an affine function $l_{+}$(which is of course, quasiconvex) such that $l_{+}(P) \leq 0$ when $P \in K$ and the equality holds only when $P \in K \cap L_{+}$. Since $L_{+}$is a two-dimensional plane without rankone connections, we can prove, as in the proof of Theorem 1.3 (by translating the plane to a two-dimensional subspace without rank-one connections) that $P_{0} \in\left(K \cap L_{+}\right)_{q, 1}$. Therefore $P_{0} \in K_{q, 2}$. The case for points on $K \cap L_{-}$is similar.

Next we prove that if $P_{0} \in\{P \in K$, $\operatorname{det} P=1\} \backslash\left(L_{+} \cup L_{-}\right)$, then $P_{0} \in K_{q, 3}$. Let

$$
f_{1}(P)=1-\operatorname{det} P
$$

for $P \in M^{2 \times 2}$. we see that $f_{1}$ is quasiconvex and if we let

$$
K_{1}=\left\{A \in K, f_{1}(A)=\sup _{X \in K} f_{1}(X)\right\}
$$

we see that

$$
K_{1}=\{P \in K, \operatorname{det} P=1\}, \quad \text { and } \sup _{X \in K} f_{1}(X)=0 .
$$


Now suppose $P_{0} \in K_{+}$, we use Šverák's quasiconvex function

$$
f_{2}(P)= \begin{cases}\operatorname{det} P, & P=P^{T}, \\ 0, & \text { otherwise. }\end{cases}
$$

We have

$$
f_{2}(P)= \begin{cases}1, & \text { if } P \in K_{+} \cap\{P \in K, \operatorname{det} P=1\}, \\ 0, & \text { if } P \in K_{-} \cap\{P \in K, \operatorname{det} P=1\} .\end{cases}
$$

Therefore $K_{2}=K_{+} \cap\{P \in K$, $\operatorname{det} P=1\}$, where

$$
K_{2}=\left\{A \in K_{1}, f_{2}(A)=\sup _{X \in K_{1}} f_{2}(X)\right\} .
$$

Finally, since $K_{2}$ is a strictly convex surface, therefore the tangent plane of $K_{2}$ passing through $P_{0}$ intersects $K_{2}$ only at $P_{0}$. Therefore there is an affine function $f_{3}$, such that $f_{3}(P) \leq 0$ on $K_{2}$ with equality holds only at $P=P_{0}$. Hence $P_{0} \in K_{q, 3}$.

If $P_{0} \in K_{-} \cap\{P \in K$, det $P=1\}$, we notice that we can replace $f_{2}$ above by

$$
f_{-}(P)=\left\{\begin{array}{l}
\operatorname{det} P, \quad P=P^{T}, \quad P \text { is negative definite, } \\
0, \quad \text { otherwise }
\end{array}\right.
$$

and finish the proof in a similar way.

Proof of Proposition 1.4. We prove the result in three simple steps.

Step 1. We show that $C\left(K_{t}\right)=C(K)_{t}$, hence $\left[C(K)_{t}\right]_{e}=\left[K_{t}\right]_{e}$ for every $t \geq 0$. Since $K \subset C(K)$, we see that $K_{t} \subset C(K)_{t}$ so that $C\left(K_{t}\right) \subset C(K)_{t}$ because $C(K)_{t}$ is convex. Let $P_{0} \in C(K)_{t}$. There is $A \in C(K)$ such that $\operatorname{dist}\left(P_{0}, C(K)\right)=\left|P_{0}-A\right| \leq t$. Carathéodory's theorem implies that there are $B_{k} \in K$, and $\lambda_{k} \geq 0$, $k=1,2, \cdots, n+1$, such that

$$
\sum_{k=1}^{n+1} \lambda_{k}=1, \quad A=\sum_{k=1}^{n+1} \lambda_{k} B_{k}
$$

Therefore,

$$
P_{0}=\left(P_{0}-A\right)+\sum_{k=1}^{n+1} \lambda_{k} B_{k}=\sum_{k=1}^{n+1} \lambda_{k}\left[\left(P_{0}-A\right)+B_{k}\right]
$$

Since $B_{k} \in K$ and $\left|P_{0}-A\right| \leq t$, we have

$$
t \geq\left|P_{0}-A\right|=\left|\left(P_{0}-A+B_{k}\right)-B_{k}\right| \geq \operatorname{dist}\left(P_{0}-A+B_{k}, K\right),
$$

$P_{0}-A+B_{k} \in K_{t}$ so that $P_{0} \in C(K)_{t}$.

Step 2. $\left[K_{t}\right]_{e} \subset \tilde{K}_{t}$. Let $P_{0} \in\left[K_{t}\right]_{e}=\left[\left(C(K)_{t}\right]_{e}\right.$. We see that $P_{0} \in \partial\left[\left(C(K)_{t}\right] \cap \partial\left[K_{t}\right]\right.$. We see that $\operatorname{dist}\left(P_{0}, C(K)\right)=\operatorname{dist}\left(P_{0}, K\right)=t$, hence $P_{0} \in \tilde{K}_{t}$.

Step 3. $\tilde{K}_{t} \subset\left(\tilde{K}_{t}\right)_{e}$. Let $P_{0} \in \tilde{K}_{t}$. If $P_{0}$ is not a convex extreme point, there are $A_{0}, B_{0} \in \tilde{K}_{t}$ and $0<\lambda<1$, such that $P_{0}=\lambda A_{0}+(1-\lambda) B_{0}$. Since $\operatorname{dist}(\cdot, C(K))$ is a convex function and

$$
\operatorname{dist}\left(\lambda A_{0}+(1-\lambda) B_{0}, C(K)\right)=\operatorname{dist}\left(A_{0}, C(K)\right)=\operatorname{dist}\left(B_{0}, C(K)\right)=t,
$$

we see that $\operatorname{dist}\left(s A_{0}+(1-s) B_{0}, C(K)\right)=t$ for every $s \in[0,1]$. 
Now, from the nearst-point property of compact convex sets and the definition of $\tilde{K}_{t}$, there are $P, A, B \in K$, such that

$$
\begin{aligned}
& \operatorname{dist}\left(P_{0}, K\right)=\operatorname{dist}\left(P_{0}, C(K)\right)=\left|P_{0}-P\right|=t, \\
& \operatorname{dist}\left(A_{0}, K\right)=\operatorname{dist}\left(A_{0}, C(K)\right)=\left|A_{0}-A\right|=t, \\
& \operatorname{dist}\left(B_{0}, K\right)=\operatorname{dist}\left(B_{0}, C(K)\right)=\left|B_{0}-B\right|=t .
\end{aligned}
$$

We can easily see that $P, A, B$ are three distinct points. We further claim that $P$ is a convex combination of $A$ and $B$ so that $P$ is not an extreme point of $K$. This contradicts $K=K_{e}$. In fact, if $P, A$ and $B$ forms a non-degenerate triangle, let us consider the orthogonal projection of the line sigment

$$
L_{0}=\left\{s A_{0}+(1-s) B_{0}, 0 \leq s \leq 1\right\}
$$

to the two-dimensional plane formed by the triangle. Therefore the distance between the three projected points from $A_{0}, B_{0}$ and $P_{0}$ to the $A, B$ and $P$ are the same. This easily leads to a contradiction in Euclidean geometry. If $A, B$ and $P$ lie in a straight line $L$, we claim that $L$ is paralell to $L_{0}$ so that $P$ is not an end-point of the line sigment formed by these three points. This is easy to see, hence $P$ is a convex comination of $A$ and $B$. The proof is complete.

Proof of Proposition 1.5. Let us rewrite the functions $F$ and $f$. We denote the orthogonal complement of $\operatorname{span}\left[E_{1}\right]$ as $E_{1}^{\perp}$, and let $P_{E_{1}}$ and $P_{E_{1}^{\perp}}$ be the orthogonal projection to $\operatorname{span}\left[E_{1}\right]$ and $E_{1}^{\perp}$ respectively.

$$
\begin{aligned}
F(P) & =\operatorname{dist}^{2}(P, K)=\min \left\{\left|P+\frac{1}{\sqrt{2}} I\right|^{2},\left|P-\frac{1}{\sqrt{2}} I\right|^{2}\right\}=\min \left\{\left|P_{E_{1}}(P)+E_{1}\right|^{2},\left|P_{E_{1}}(P)-E_{1}\right|^{2}\right\}+\left|P_{E_{1}^{\perp}}(P)\right|^{2} \\
& =\min \left\{|t+1|^{2},|t-1|^{2}\right\}+s^{2}+x^{2}+y^{2} .
\end{aligned}
$$

Here we have used the notition $P=t E_{1}+s E_{2}+x E_{3}+y E_{4}$. It is not difficult to see that we can write $f=Q F$ as

where

$$
f(P)=g(t)-t^{2}+s^{2}+x^{2}+y^{2}
$$

$$
g(t)=\left\{\begin{aligned}
\frac{1}{2}, & \text { if }|t| \leq \frac{1}{2} \\
(t-1)^{2}+t^{2}, & \text { if } t \geq \frac{1}{2} \\
(t+1)^{2}+t^{2}, & \text { if } t \leq-\frac{1}{2} .
\end{aligned}\right.
$$

We see that $f(P)=F(P)$ if $|t|>1 / 2$. Notice that $g(t)$ is locally strictly convex if $|t|>1 / 2$. So we can write $f$ in another form

$$
f(P)=g(t)+2 s^{2}+\left(x^{2}+y^{2}-t^{2}-s^{2}\right)=g(t)+2 s^{2}-2 \operatorname{det} P=G(P)-\operatorname{det} P,
$$

where $G(P)=g(t)+2 s^{2}$. We see that $f$ is the sum of a convex function $G$ and a quasiconvex function (null lagrangian) $-\operatorname{det} P$. We then can use the idea in the proof of Theorem 1.2.

Proof of (1). Suppose $P_{0} \in M_{1}$ and $\left|t_{0}\right|>1 / 2$, we let

$$
h(P)=g\left(t_{0}\right)+g^{\prime}\left(t_{0}\right)\left(t-t_{0}\right)+2 s_{0}^{2}+4 s_{0}\left(s-s_{0}\right)
$$

We see that $h$ is an affine function and $G(P) \geq h(P)$ and the equality holds in $K_{f\left(P_{0}\right)}$ if and only if $t=t_{0}$, $s=s_{0}$. Let

we see that $K_{1}=\partial K_{f\left(P_{0}\right)}$.

$$
K_{1}=\left\{P, f(P)=\sup \left\{f(A), A \in K_{f\left(P_{0}\right)}\right\}\right\}
$$


Let

$$
K_{2}=\left\{P, h(P)=\sup \left\{h(A), A \in K_{1}\right\}\right\}
$$

we have

$$
K_{2}=\left\{P, s=s_{0}, t=t_{0}, P \in K_{1}\right\}
$$

so that $f\left(P_{0}\right)=f(P), t=t_{0}, s=s_{0}$, hence $x^{2}+y^{2}=x_{0}^{2}+y_{0}^{2}$. We obtain

$$
K_{2}=\left\{P \in M^{2 \times 2}, t=t_{0}, s=s_{0}, x^{2}+y^{2}=x_{0}^{2}+y_{0}^{2}\right\} .
$$

Since $\left(x_{0}, y_{0}\right)$ is on a circle, we see that $P_{0} \in\left(K_{2}\right)_{q, 1}$, hence $P_{0} \in\left(K_{f\left(P_{0}\right)}\right)_{q, 3}$.

The case $\left|t_{0}\right|=1 / 2$ will be dealt with in Part (2) and Part (3).

Proof of (2). If $P_{0} \in M_{2}=\left\{P_{0},\left|t_{0}\right|<1 / 2, t_{0}^{2}>x_{0}^{2}+y_{0}^{2}\right\}, G(\cdot)$ is degenate when $P \in M_{2}$. We consider

$$
h(P)=g\left(t_{0}\right)+2 s_{0}^{2}\left(s-s_{0}\right) .
$$

We see that $G(P) \geq h(P)$ in $K_{f\left(P_{0}\right)}$ and the equality hold only when $|t| \leq 1 / 2$ and $s=s_{0}$. If we let

$$
K_{1}=\left\{P, f(P)=\sup \left\{f(A), A \in K_{f\left(P_{0}\right)}\right\}\right\},
$$

as in the proof of Part (1), we have $K_{1}=\partial K_{f\left(P_{0}\right)}$.

Let

$$
K_{2}=\left\{P, h(P)=\sup \left\{h(A), A \in K_{1}\right\}\right\},
$$

we have $s=s_{0},|t| \leq 1 / 2$ and $f(P)=f\left(P_{0}\right)$. Since $g(t)=1 / 2$ when $|t| \leq 1 / 2$. These restrictions give

$$
\frac{1}{2}-t_{0}^{2}+s_{0}^{2}+x_{0}^{2}+y_{0}^{2}=\frac{1}{2}-t^{2}+s_{0}^{2}+x^{2}+y^{2}
$$

with $|t|<1 / 2$. Hence we have

$$
K_{2}=\left\{P \in M^{2 \times 2}, t^{2}=x^{2}+y^{2}+\left(t_{0}^{2}-x_{0}^{2}+y_{0}^{2}\right), s=s_{0},|t|<\frac{1}{2}\right\} .
$$

New we write $P \in K_{2}$ as

$$
P=t E_{1}+s_{0} E_{2}+x E_{3}+y E_{4}=\frac{1}{\sqrt{2}}\left(\begin{array}{cc}
t+x & y \\
y & t-x
\end{array}\right)+s_{0} E_{2},
$$

and we obtain

$$
\operatorname{det}\left(P-s_{0} E\right)=t^{2}-x^{2}-y^{2}=t_{0}^{2}-x_{0}^{2}-y_{0}^{2} .
$$

We see that $K_{2}$ is a translation of the set of symetric $2 \times 2$ matrices in Theorem 1.5 and Remark 1.4. In fact, if we define $\tilde{f}_{ \pm}(P)=f_{ \pm}\left(P-s_{0} E_{2}\right)$, where $f_{ \pm}$are the quasiconvex function constructed by Šverák ( $c f$. proof of Th. 1.5), we see that $P_{0} \in\left(K_{2}\right)_{q, 2}$. Therefore $P_{0} \in\left(K_{f\left(P_{0}\right)}\right)_{q, 4}$.

If $\left|t_{0}\right|=1 / 2$ and $t_{0}^{2}>x_{0}^{2}+y_{0}^{2}$, then $P_{0}$ is on the boundary of $K_{2}$ as a two-dimensional manifold. We see that $P_{0}$ then is an convex exposed point of $K_{2}$, hence $P_{0} \in\left(K_{2}\right)_{q, 1}$, so $P_{0} \in\left(K_{f\left(P_{0}\right)}\right)_{q, 3}$.

Proof of (3). When $P_{0} \in M_{3}$, we may conclude that $P_{0} \notin\left(K_{f\left(P_{0}\right)}\right)_{q, e}$ if we can show that $P_{0}$ is a convex combination of two rank-one connected matrices in $K_{f\left(P_{0}\right)}$. We can restrict our search in $K_{2} \subset K_{f\left(P_{0}\right)}$ used in the proof of Part (2):

$$
K_{2}=\left\{P \in M^{2 \times 2}, t^{2}=x^{2}+y^{2}+\left(t_{0}^{2}-x_{0}^{2}+y_{0}^{2}\right), s=s_{0},|t|<\frac{1}{2}\right\} .
$$


However, this time, we have $t_{0}^{2}-x_{0}^{2}-y_{0}^{2} \leq 0$. Therefore $K_{2}$ is part of a one sheet hyperbola

$$
t^{2}=x^{2}+y^{2}+\left(t_{0}^{2}-x_{0}^{2}+y_{0}^{2}\right), s=s_{0},|t|<\frac{1}{2},
$$

if $t_{0}^{2}-x_{0}^{2}-y_{0}^{2}<0$. In this case It is easy to see that the two lines on the hyperbola passing through are rank-one connected lines in the sense that any two points on one line are rank-one connected. Therefore $P_{0}$ is a convex combination of two rank-one connected points in $K_{2}$.

If $t_{0}^{2}-x_{0}^{2}-y_{0}^{2}=0, K_{2}$ is part of a symetric cone, there is a line passing through $P_{0}$ which is rank-one connected. Therefore we can conclude, in both cases that $P_{0}$ is not a quasiconvex extreme point of $K_{f\left(P_{0}\right)}$.

Let us complete the the case $\left|t_{0}\right|=1 / 2$ for Part (1) when $t_{0}^{2} \leq x_{0}^{2}+y_{0}^{2}$. We see as in the proof of Part (2) that $P_{0}$ is on the boundary of $K_{2}$ as a two-dimensional surface. Hence $P_{0}$ is an convex exposed point of $K_{2}$. Thus $P_{0} \in\left(K_{f\left(P_{0}\right)}\right)_{q, 3}$.

Proof of Part (4). When $f(P) \leq 1 / 4$, we will see that $|t| \geq 1 / 2$. For $|t|<1 / 2$,

$$
f(P)=\frac{1}{2}-t^{2}+s^{2}+x^{2}+y^{2} \geq \frac{1}{2}-t^{2}>\frac{1}{4} .
$$

Therefore $P \in M_{1}$.

Proof of (5). If we take $P(t)=t E_{1}$, and let $t \geq 1 / 2$ we see that $f(P(t))=g(t)-t^{2}=(t-1 \mid)^{2}$. Therefore $M_{1} \cap K_{\alpha} \neq \emptyset$.

If we take $P(t, s)=t E_{1}+s E_{2}$ for $|t|<1 / 2$ and $s \in \mathbb{R}$, we see that

$$
\inf \{f(P(t, s)),|t|<1 / 2, s \in \mathbb{R}\}=1 / 4
$$

and $f(P(t)=1 / 4$ when $t= \pm 1 / 2$.

$$
\sup \{f(P(t, s)),|t|<1 / 2, s \in \mathbb{R}\}=\infty .
$$

Also, since $x=y=0$, when $t \neq 0,|t|<1 / 2, P(t, s) \in M_{2}$. We conclude that $K_{\alpha} \cap M_{2} \neq \emptyset$, when $\alpha>1 / 4$.

If $P \in M_{3}, t^{2} \leq x^{2}+y^{2}$, and $|t|<1$,

$$
f(P)=\frac{1}{2}+s^{2}+\left(x^{2}+y^{2}-t^{2}\right) \geq \frac{1}{2} .
$$

Let us take $t_{0}=1 / 4, x_{0}=1 /(4 \sqrt{2}), y_{0}=1 /(4 \sqrt{2})$. We have $t_{0}^{2}=x_{0}^{2}+y_{0}^{2}$. Therefore, if we let $P_{s}$ $=t_{0} E_{1}+s E_{2}+x_{0} E_{3}+y_{0} E_{4}$, with $s \in \mathbb{R}$, we have $P_{s} \in M_{3}$ and $f\left(P_{s}\right)=\frac{1}{2}+s^{2}$. Hence $M_{3} \cap K_{\alpha} \neq \emptyset$ as $\alpha \geq 1 / 2$.

Proof of Part (6). We have in general $f(P) \leq F(P)$ for $P \in M^{2 \times 2}$. We can also see that $f(P)=F(P)$ when $|t| \geq 1 / 2$. Now if $|t|<1 / 2, P \in M_{2} \cup M_{3}$ and we let $0 \leq t<1 / 2$, then

$$
g(t)-t^{2}=\frac{1}{2}-t^{2}<(t-1)^{2} \leq(t+1)^{2} .
$$

Similarly, if $-1 / 2<t \leq 0$

We have

$$
g(t)-t^{2}=\frac{1}{2}-t^{2}<(t+1)^{2} \leq(t-1)^{2} .
$$

$$
f(P)=\frac{1}{2}-t^{2}+s^{2}+x^{2}+y^{2}<\min \left\{(t-1)^{2},(t+1)^{2}\right\}+s^{2}+x^{2}+y^{2}=F(P) .
$$

Thus $f(P)<F(P)$ if and only if $|t|<1 / 2$. The proof is complete. 
I would like to thank Professor John M. Ball for helpful discussions on the geometric discription of quasiconvex extreme points. This work is supported by Macquarie University through Macquarie University Research Grant.

\section{REFERENCES}

[1] E.M. Alfsen, Compact Convex Sets and Boundary Integrals. Springer-Verlag (1971).

[2] E. Acerbi and N. Fusco, Semicontinuity problems in the calculus of variations. Arch. Rational Mech. Anal. 86 (1984) 125-145.

[3] H. Berliocchi and J.M. Lasry, Intégrandes normales et mesures paramétrées en calcul des variations. Bull. Soc. Math. France 101 (1973) 129-184.

[4] J.M. Ball, Convexity conditions and existence theorems in nonlinear elasticity. Arch. Rational Mech. Anal. 63 (1977) $337-403$.

[5] J.M. Ball, A version of the fundamental theorem of Young measures, in Partial Differential Equations and Continuum Models of Phase Transitions, edited by M. Rascle, D. Serre and M. Slemrod. Springer-Verlag (1989) 207-215.

[6] J.M. Ball, Sets of gradients with no rank-one connections. J. Math. Pures Appl. 69 (1990) 241-259.

[7] K. Bhattacharya, N.B. Firoozye, R.D. James and R.V. Kohn, Restrictions on Microstructures. Proc. Roy. Soc. Edinburgh Sect. A 124 (1994) 843-878.

[8] J.M. Ball and R.D. James, Fine phase mixtures as minimizers of energy. Arch. Rational Mech. Anal. 100 (1987) 13-52.

[9] J.M. Ball and R.D. James, Proposed experimental tests of a theory of fine microstructures and the two-well problem. Philos. Trans. Roy. Soc. London Ser. A 338 (1992) 389-450.

[10] J.M. Ball and K.-W. Zhang, Lower semicontinuity and multiple integrals and the biting lemma. Proc. Roy. Soc. Edinburgh Sect. A 114 (1990) 367-379.

[11] M. Chipot and D. Kinderlehrer, Equilibrium configurations of crystals. Arch. Rational Mech. Anal. 103 (1988) $237-277$.

[12] B. Dacorogna, Direct Methods in the Calculus of Variations. Springer-Verlag (1989).

[13] B. Dacorogna and P. Marcellini, Théorème d'existence dans le cas scalaire et vectoriel pour les équations de Hamilton-Jacobi. C. R. Acad. Sci. Paris Sér. I Math. 322 (1996) 237-240.

[14] B. Dacorogna and P. Marcellini, Sur le problème de Cauchy-Dirichlet pour les systèmes d'équations non linéaires du premier ordre. C. R. Acad. Sci. Paris Sér. I Math. 323 (1996) 599-602.

[15] B. Dacorogna and P. Marcellini, General existence theorems for Hamilton-Jacobi equations in the scalar and vectorial case. Acta Math. 178 (1997) 1-37.

[16] B. Dacorogna and P. Marcellini, Cauchy-Dirichlet problem for first order nonlinear systems. J. Funct. Anal. 152 (1998) 404-446.

[17] B. Dacorogna and P. Marcellini, Implicit second order partial differential equations. Ann. Scuola. Norm. Sup. Pisa Cl. Sci. (4) 25 (1997) 299-328.

[18] J.L. Kelly, General Topology. van Nostrand (1955).

[19] D. Kinderlehrer and P. Pedregal, Characterizations of Young measures generated by gradients. Arch. Rational Mech. Anal 115 (1991) 329-365.

[20] R.V. Kohn, The relaxation of a double well energy. Cont. Mech. Therm. 3 (1991) 981-1000.

[21] S.R. Lay, Convex Sets and Their Applications. John Wiley \& Sons (1982).

[22] C.B. Morrey Jr., Multiple integrals in the calculus of variations. Springer (1966).

[23] S. Müller and V. Šverák, Attainment results for the two-well problem by convex integration. Preprint (1993).

[24] Yu.G. Reshetnak, Liouville's theorem on conformal mappings under minimal regularity assumptions. Siberian Math. J. 8 (1967) 631-653.

[25] R.T. Rockafellar, Convex Analysis. Princeton University Press (1970).

[26] W. Rudin, Functional Analysis. McGraw-Hill (1973).

[27] V. Šverák, On regularity for the Monge-Ampère equations. Preprint.

[28] V. Šverák, New examples of quasiconvex functions. Arch. Rational Mech. Anal. 119 (1992) 293-330.

[29] V. Šverák, On the problem of two wells, in Microstructure and phase transitions, edited by D. Kinderlehrer, R.D. James, M. Luskin and J. Ericksen. Springer, IMA J. Appl. Math. 54 (1993) 183-189.

[30] V. Šverák, On Tartar's conjecture. Ann. Inst. H. Poincaré 10 (1993) 405-412.

[31] L. Tartar, Compensated compactness and applications to partial differential equations, in Nonlinear Analysis and Mechanics: Heriot-Watt Symposium, IV, edited by R.J. Knops. Pitman (1979).

[32] K.-W. Zhang, A construction of quasiconvex functions with linear growth at infinity. Ann. Scuola Norm. Sup. Pisa Cl. Sci. (4) XIX (1992) 313-326.

[33] K.-W. Zhang, On connected subsets of $M^{2 \times 2}$ without rank-one connections. Proc. Roy. Soc. Edinburgh Sect. A 127 (1997) $207-216$.

[34] K.-W. Zhang, On various semiconvex hulls in the calculus of variations. Calc. Var. Partial Differential Equations 6 (1998) $143-160$.

[35] K.-W. Zhang, On the structure of quasiconvex hulls. Ann. Inst. H. Poincaré Anal. Non Linéaire 15 (1998) 663-686. 